August 2019

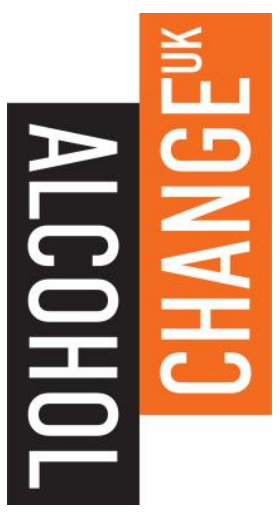

\title{
Enhancing a brief online intervention to reduce alcohol consumption
}

Prof. Paul Norman, Dr Thomas Webb, Dr Abigail Millings, University of Sheffield, and Ms Laura Pechey, Haringey Advisory Group on Alcohol 


\section{Author details}

Prof. Paul Norman, University of Sheffield

Dr Thomas Webb, University of Sheffield

Dr Abigail Millings, University of Sheffield

Ms Laura Pechey, Haringey Advisory Group on Alcohol

\section{Contact person details}

Prof. Paul Norman, University of Sheffield

Department of Psychology, University of Sheffield, Cathedral Court, 1 Vicar Lane, Sheffield, S1 2LT

p.norman@sheffield.ac.uk

\section{Institutional details}

To educate others and ourselves and to learn through doing so, thereby improving the world.

\section{Acknowledgements}

We would like to thank Petra Kuunders of Orbis Media for producing the four versions of the DontBottleltUp website that were tested in the research project.

This report was funded by Alcohol Change UK. Alcohol Change UK works to significantly reduce serious alcohol harm in the UK. We create evidence-driven change by working towards five key changes: improved knowledge, better policies and regulation, shifted cultural norms, improved drinking behaviours, and more and better support and treatment.

Find out more at alcoholchange.org.uk. 


\section{Contents}

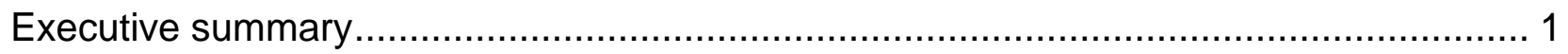

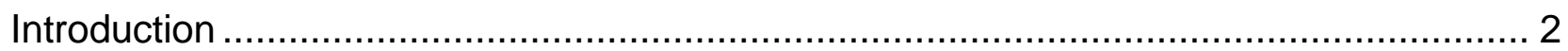

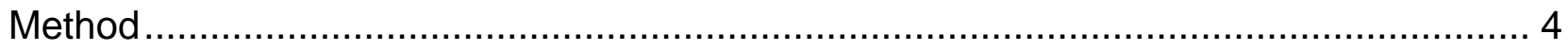

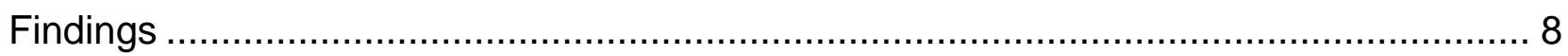

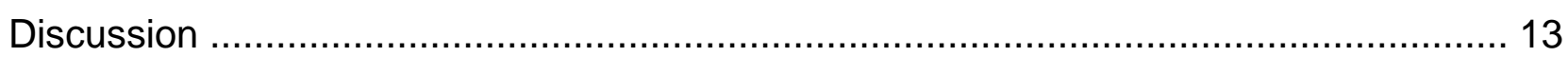

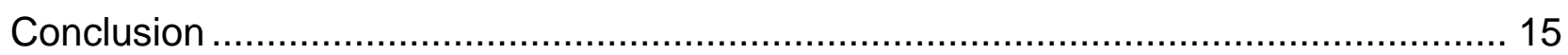

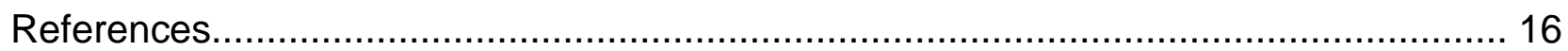

Appendix - Example screenshots of the DBIU website ....................................... 19 



\section{Executive summary}

Brief alcohol interventions that are delivered in primary care settings have been found to reduce alcohol consumption. The reach, and potential, of such interventions could be expanded considerably if delivered online. However, despite the potential reach of brief online alcohol interventions, their effectiveness may be compromised by low levels of engagement and by the inclusion of ineffective behaviour change techniques.

In this study we sought to assess (i) whether a tunnelled intervention in which the content is delivered in a pre-specified order would lead to greater engagement and effectiveness than a free-roam version in which the content can be viewed in any order and (ii) whether being instructed to form if-then plans (i.e., implementation intentions) to cut down on drinking would lead to greater reductions in alcohol consumption than only choosing strategies to cut down.

After completing baseline measures of alcohol consumption, participants (university staff and students) were randomly allocated to one of four versions of a brief online alcohol intervention (DontBottleltUp) in a 2 (structure: free-roam versus tunnelled) $\times 2$ (planning: strategies versus if-then plans) factorial design. Measures of engagement with the online intervention (pages visited, time) were recorded automatically. Participants' alcohol consumption was assessed again one and six months later.

The structure of the online intervention had a significant effect on engagement.

Participants who viewed the tunnelled version viewed significantly more pages and spent significantly more time on the website than participants who viewed the free-roam version. Significant reductions in alcohol consumption were observed between baseline and both follow-up time points; however, neither the structure of the intervention nor the type of planning had a significant effect on reductions in alcohol consumption.

The present results indicate that brief online alcohol interventions should employ a tunnelled structure in order to increase engagement. However, given that neither the structure of the intervention nor the type of planning influenced effectiveness, future experimental work is needed to identify the "active ingredients" (i.e., key behaviour change techniques) of brief online alcohol interventions. 
Brief alcohol interventions, typically comprising a single session (5-25 minutes) delivered opportunistically by a health professional in a primary care setting, have been found to lead to small but significant reductions in alcohol consumption. Moyer et al. (2002) reported an average effect size of $d=0.26$ at 6-12 months follow-up, while Bertholet et al. (2005) and Kaner et al. (2005) reported average reductions in weekly alcohol consumption of approximately $40 \mathrm{~g}$ of ethanol (= 5 units). The reach, and potential, of such interventions could be expanded considerably if delivered online. Brief online alcohol interventions are likely to be low-cost, easy to deliver, and convenient to use. However, online interventions have been found to have smaller, but still significant, effects on alcohol consumption $(d=0.14)$ than those delivered in person (Webb et al., 2010). Research is therefore needed to enhance the effectiveness of current online interventions to reduce alcohol consumption.

Engagement with online interventions is often low (Kelders et al., 2012) which, in turn, is likely to reduce their effectiveness (Donkin et al., 2011; Manwaring et al., 2008). The way in which the content is delivered in an online intervention may have an important impact on levels of engagement. One key distinction is between free-roam structures that allow users to view the intervention content in any order and tunnelled structures that guide users through the intervention content in a specific, pre-determined, order (Fogg, 2003). Free-roam online interventions provide users with more control and autonomy over what content they view and in which order and, as a result, should increase engagement. In addition, users can focus on the most relevant material, which may increase effectiveness. However, the increased autonomy may lead to users not viewing the most important material and/or choosing not to view any (or very little) material. Tunnelled online interventions may therefore help to ensure that users are directed to the most important material and/or view material that would otherwise not be viewed. However, the resultant lack of control and autonomy may lead to a reactance effect (e.g., frustration) and drop-out, thereby reducing engagement.

Systematic reviews of online interventions have mainly reported null findings on the impact of the structure on engagement and effectiveness. For example, Kelders et al. (2012) in their systematic review of online health interventions found no effect of the structure of the intervention (i.e., tunnelled versus free-roam) on engagement or effectiveness, although many of the interventions included in the review were modular rather than brief interventions. In addition, Fredericks et al. (2012) in their systematic review of online educational interventions for self-care behaviours following cardiac surgery concluded that most tunnelled interventions had minimal effectiveness. In contrast, free-roam interventions were associated with increases in self-care behaviours, although it should be noted that only three such interventions were included in the review. Few studies though have directly compared free-roam versus tunnelled versions of the same intervention in a randomised controlled trial. Crutzen et al. (2012) found that participants who viewed a tunnelled version of a 12 page information website about hepatitis visited more pages, spent more time on the website, and had greater knowledge about hepatitis at one-month follow-up, than participants who viewed a free-roam version. Similarly, McClure et al. (2013) reported that participants who had access to the tunnelled version of an online smoking cessation intervention visited more pages and spent more time online than those who had access to the free-roam version. However, McClure et al. (2014) found that the structure of the online smoking cessation intervention had no impact on cessation rates at 12-month follow-up. To date, no studies have tested the effect of manipulating the structure of online alcohol interventions on engagement and effectiveness. 
To be effective, brief online alcohol interventions need to include behaviour change techniques that have been found to influence alcohol consumption. Brief alcohol interventions typically provide people with tips, or strategies, on how to reduce consumption. These tips typically take the form of lists of behavioural strategies (e.g., avoid drinking in rounds) taken from the Australian "Drink-Less" programme (Gomel et al., 1994) and routinely incorporated into NHS material on alcohol (e.g., Simple Structured Advice Intervention Tool). However, such strategies may only be effective when they are linked to specific high-risk situations (e.g., when out with heavy drinking friends). Meta-analytic reviews have found that instructing people to form if-then plans (i.e., implementation intentions) that explicitly link high-risk situations with appropriate strategies has a medium-to-large sized effect on health-related behaviour (Gollwitzer and Sheeran, 2006).

To date, few studies have directly compared the effects of forming if-then plans versus simply considering strategies for cutting down on alcohol consumption. In a series of small-scale studies (Arden and Armitage, 2012; Armitage, 2015; Armitage and Arden, 2012) participants were presented with a list of high-risk situations for heavy episodic drinking and potential strategies for reducing alcohol consumption. Greater reductions in alcohol consumption were found among those who were instructed to link situations and strategies than among those who were instructed to simply tick relevant situations and strategies (without linking them). However, despite some encouraging findings, these studies suffer from a number of limitations. First, the sample sizes were very small (approximately 20-30 participants per condition) which increases the likelihood of both Type 1 and Type 2 errors (false positives and false negatives). Second, whereas the situations were taken from a scale designed to identify high-risk situations for heavy episodic drinking (Maddock et al., 2000), the strategies were based on a measure of ten processes of change that are important in the treatment of alcohol dependency (CPRC, 2010). Some of these strategies are unlikely to be relevant for non-dependent and/or non-treatment samples (e.g., "I will stop to think about how my drinking is hurting people around me"). Third, the interventions were all embedded in a questionnaire on beliefs about alcohol or binge drinking, rather than being an explicit component of an intervention or advice sheet. Finally, the questionnaires were delivered in person rather than online. Therefore, there is a need to test the effect of forming if-then plans versus simply considering strategies in an alcohol intervention that is delivered online and includes strategies that may be more relevant to non-dependent drinkers (i.e., as commonly used in current alcohol advice materials).

The present study therefore assessed the impact of two enhancements to a brief online alcohol intervention (www.dontbottleitup.org.uk). First, the study compared the impact of a free-roam versus a tunnelled structure on engagement as well as alcohol consumption. Second, the study compared the effectiveness of instructing users to choose strategies to cut down on their drinking versus making if-then plans linking high-risk situations with these potential strategies on subsequent alcohol consumption. 


\section{Method}

\section{Procedure and design}

A recruitment email was sent to a university staff and student volunteers list, inviting them to take part in an online study on alcohol feedback and advice. The email contained a link to further information about the study and a consent form. After providing informed consent, participants were directed to the baseline questionnaire which contained measures of demographics and typical alcohol consumption. At the end of the questionnaire, participants were instructed to click on a link to the DontBottleltUp (DBIU) website, at which point they were randomly allocated to one of four versions of the website using a 2 (structure: free-roam versus tunnelled) $\times 2$ (planning: strategies versus if-then plans) factorial design. Within the DBIU website, all participants completed the AUDIT measure (Babor et al., 2001) and then received feedback on their risk level (i.e., lower risk, increasing risk, higher risk, high risk/possibly dependent). Participants then had access to 4-5 pages of information on alcohol (tailored to their risk level) that was either tunnelled or free-roam. For increasing risk and higher risk drinkers, the information included a page on how to cut down on drinking with instructions to either choose strategies or form if-then plans linking high-risk situations with the strategies. Information on engagement with the website (e.g., pages visited, time spent on the website) was automatically recorded by a back-end data log and linked to the baseline survey data using unique ID codes. An email with a link to a follow-up questionnaire to assess subsequent alcohol consumption was sent to participants one and six months later. Up to two reminder emails were sent to increase response rates to the follow-up questionnaires.

The study was approved by the Department of Psychology Research Ethics Committee in line with the University of Sheffield's Research Ethics Approval Procedure.

Participation in the study was voluntary but was incentivised by the opportunity to be entered into a prize draw to win one of three $£ 50$ gift vouchers at each time point.

\section{Measures}

\section{Demographics}

The baseline questionnaire contained questions on age, gender, ethnicity, nationality and staff/student status.

\section{Alcohol consumption}

Typical alcohol consumption at baseline was assessed using a modified version of the Alcohol Outcomes Record taken form the Treatment Outcomes Profile (Public Health England, 2017). In line with an alcohol timeline follow-back method (Sobell and Sobell, 1992), participants were asked to report what they drank on each day of the week during a typical week. They were presented with a table that contained a list (in rows) of 15 common drinks (e.g., pint of ordinary strength lager, beer or cider; large glass of wine $(250 \mathrm{ml})$, single measure (shot) of spirits $(25 \mathrm{ml}) ; 1$ litre bottle of strong cider) and spaces to type in how many of each type of drink they typically drank on each day of the week (that were listed as column headings). The table also contained three rows for participants to type in other drinks not listed in the table. The drinks were converted into 
units of alcohol using values listed on the Alcohol Outcomes Record form. Other drinks were coded and converted into units using an online unit calculator (Drinkaware, 2017). The number of units consumed in a typical week was computed as well as the frequency of binge drinking (i.e., number of days when females/males consumed $6 / 8$ or more units of alcohol, respectively). The same procedure was used to assess alcohol consumption in the one- and six-month follow-up questionnaires, except that participants were instructed to think about what they typically drank on each day of the week over the previous month.

\section{AUDIT}

Participants completed the 10-item Alcohol Use Disorders Identification Test (AUDIT; Babor et al., 2001) as part of the DBIU website. The AUDIT is a widely used screening tool for identifying hazardous and harmful patterns of alcohol consumption. Scores on the AUDIT can range between 0 and 40. Respondents with scores between 0-7 are categorised as lower risk drinkers, between 8-15 as increasing risk drinkers, between 1619 as higher risk drinkers and 20 or above as high risk/possibly dependent drinkers. Participants completed the AUDIT measure at baseline and at six-month follow-up.

\section{Engagement}

A back-end data log automatically logged which pages each user visited and the total time spent on the DBIU website (from completing the AUDIT measure to their last click on the website).

\section{Experimental conditions}

\section{Structure: Tunnelled versus free-roam}

After receiving feedback on their risk score, participants randomly allocated to the tunnelled version of the DBIU website were instructed to click on an arrow to move to the next page (and so on) so that they moved sequentially through the pages on the website. Participants had the opportunity to view 4-5 pages of information about alcohol (tailored to their risk level) that they could view in a pre-determined order. The titles of the pages for each risk group are listed in Table 1.

After receiving feedback on their risk score, participants randomly allocated to the freeroam version of the DBIU website were presented with a table of options (that were tailored by risk level) for them to click and read as many or as few pages as they wished, in any order.

Screenshots of pages from the DBIU website are provided in Appendix A.

\section{Planning: Strategies versus if-then plans}

Increasing risk and higher risk drinkers randomly allocated to the strategies condition were presented with a list of ten strategies for cutting down on drinking (e.g., Avoid drinking in rounds or in large groups) taken from the Australian "Drink-Less" programme (Gomel et al., 1994) and also used in NHS alcohol information leaflets (e.g. Simple Structured Advice Intervention Tool). Participants were instructed to drag up to three 
strategies (and/or write their own strategies) into three boxes to form strategies to cut down. A screenshot of the strategies and instructions is provided in Appendix A.

Increasing risk and higher risk drinkers randomly allocated to the if-then planning condition were presented with a list of ten high-risk situations taken from research on the situations in which people are typically tempted to engage in heavy episodic drinking (e.g., If I am out with others who are drinking a lot) (Maddock et al., 2000) as well as the ten strategies for cutting down. Participants were instructed to form up to three if-then plans to cut down by dragging the situations and strategies into adjacent boxes (e.g. If I am out with others who are drinking a lot, then I will set myself a limit and stick to it) and/or by writing their own if-then plans. A screenshot of the planning exercise is provided in Appendix A.

Table 1. Alcohol information pages by risk level

\begin{tabular}{|c|c|c|c|c|}
\hline & \multicolumn{4}{|c|}{ Risk Level } \\
\hline & Lower & Increasing & Higher & $\begin{array}{l}\text { High/Possibly } \\
\text { Dependent }\end{array}$ \\
\hline What do units mean? & $x$ & $x$ & $x$ & $x$ \\
\hline $\begin{array}{l}\text { How does my drinking } \\
\text { compare? }\end{array}$ & $X$ & $\mathrm{X}$ & $X$ & $x$ \\
\hline What are the risks? & $x$ & $x$ & $x$ & $x$ \\
\hline What can I do next? & $x$ & & & \\
\hline Am I an alcoholic? & & & $\mathrm{X}$ & $x$ \\
\hline Create a plan & & $x$ & $x$ & \\
\hline Plan your next step & & & & $x$ \\
\hline
\end{tabular}

\section{Data analysis}

Data were examined for outliers. Participants who reported weekly alcohol consumption values (units) > 3 SD from the mean were removed from the dataset. In addition, participants who had website-visit times $>3$ SD from the mean were excluded from the analyses examining time spent on the website. 
Randomisation checks were conducted by comparing the four conditions on baseline measures of gender, staff/student status, ethnicity, and nationality using chi-square tests and on baseline measures of age, weekly alcohol consumption (units), frequency of binge drinking and AUDIT scores using one-way between-participants ANOVAs.

The effect of the structure of the DBIU website on the number of pages visited and time spent on the website was tested using two-way between-participants ANOVAs with structure and risk level as fixed factors (to control for the fact that the different risk groups could view different numbers of pages). Chi-square tests were also used to compare the number of participants in each structure condition who viewed specific pages on the website.

The effect of the structure of the DBIU website and the type of planning on alcohol consumption between baseline and one- and six-month follow-up was tested using mixed-measures ANOVAs with structure and planning as the between-participants factors and time as the repeated measures factor. The dependent variables were weekly consumption of alcohol (units) and frequency of binge drinking assessed at baseline and at one-month and six-month follow-up.

At each follow-up time point, attrition analyses were conducted by comparing those who were lost to follow-up to those who completed both questionnaires by condition and on baseline measures of demographics and alcohol consumption, using chi-square tests and independent samples t-tests as appropriate. 


\section{Findings}

\section{Participants}

In total, 580 staff and students clicked the link to further information about the study. Of these, 10 did not consent to take part in the study, 137 did not complete the baseline measures and 143 did not complete the AUDIT measure in the DBIU website and so received no feedback or information on their alcohol consumption. A further four participants were subsequently excluded due to extreme baseline weekly alcohol consumption (> 3 SDs above the mean), resulting in a final baseline sample of 286 participants of whom 105 were university staff and 181 were university students.

The sample comprised 102 males and 180 females (other $n=4$ ) with a mean age of 27.63 years $(S D=10.00)$. The majority of the sample were UK nationals $(76.9 \%)$ and described their ethnicity as White (85.7\%). The sample consumed a mean of 16.47 (SD = 17.67) units per week at baseline and engaged in binge drinking a mean of 0.92 (SD = 1.26) times per week. The mean AUDIT score was $9.45(S D=5.96)$ and the sample contained 106 lower risk drinkers, 133 increasing risk drinkers, 26 higher risk drinkers and 21 high risk/possibly dependent drinkers.

Of the baseline sample, 241 participants also completed the one-month follow-up questionnaire, although five participants were subsequently excluded due to extreme weekly alcohol consumption ( $>3$ SDs above the mean), resulting in a final sample of 236 participants at one-month follow-up. In addition, 228 participants completed the six-month follow-up questionnaire, although four participants were subsequently excluded due to extreme weekly alcohol consumption, resulting in a final sample of 224 participants at six-month follow-up.

\section{Randomisation checks}

Of the final sample, 75 were randomly allocated to the free-roam structure + if-then plans condition, 67 to the free-roam structure + strategies condition, 72 to the tunnelled structure + if-then plans condition, and 72 to the tunnelled structure + strategies condition. Randomisation checks revealed no significant differences between the conditions on staff/student status, $X^{2}(3, N=286)=0.07, p=.996$, gender, $X^{2}(3, N=282)$ $=0.42, p=.94$, age, $F(3,282)=0.38, p=.77$, nationality, $X^{2}(3, N=286)=2.39, p=.50$, ethnicity, $X^{2}(3, N=286)=1.09, p=.78$, weekly alcohol consumption (units), $F(3,282)=$ $1.48, p=.22$, frequency of binge drinking, $F(3,282)=1.41, p=.24$, and AUDIT score, $F(3,282)=0.58, p=.63$.

\section{Engagement}

The structure of the intervention had a significant effect on the number of pages visited on the DBIU website, $F(1,278)=75.80, p<.001, d=1.36$; participants who received the tunnelled version viewed more pages $(M=3.49, S D=1.52)$ than participants who received the free-roam version $(M=1.37, S D=1.59)$. The main effect of risk level, 
$\mathrm{F}(3,278)=0.27, \mathrm{p}=.85$, and the interaction between risk level and structure condition, $F(3,278)=0.56, p=.64$, were non-significant.

The structure of the intervention also had a significant effect on the amount of time spent on the DBIU website, $F(1,275)=4.63, p=.03, d=0.22$; participants who received the tunnelled version spent more time (seconds) on the website $(M=200.48, S D=123.31$ ) than participants who received the free-roam version $(M=173.49, S D=119.28)$. The main effect of risk level was also significant, $F(3,275)=3.15, p=.02$; post-hoc tests revealed that lower risk drinkers spent less time on the website $(M=158.69, S D=$ 114.20) than increasing risk $(M=197.85, S D=121.31)$, higher risk $(M=215.54, S D=$ $118.72)$ and high risk/possibly dependent $(M=230.65, S D=143.99)$ drinkers. The interaction between risk level and structure condition, $F(3,275)=0.90, p=.44$, was nonsignificant.

As shown in Table 2, participants who received the tunnelled version were more likely to view each of the specific pages contained in the DBIU website than participants who received the free-roam version. In particular, participants were more likely to view specific pages providing information on the meaning of units, $X^{2}(1, N=286)=39.28, p<.001$, comparisons with national data on the prevalence of risk levels, $X^{2}(1, N=286)=86.19, p$ $<.001$, and the risks of drinking alcohol, $X^{2}(1, N=286)=92.14, p<.001$, than participants who received the free-roam version.

Lower risk drinkers who received the tunnelled version of the DBIU website were more likely to view the page providing advice on their drinking, $x^{2}(1, N=106)=26.85, p<.001$, than those who received the free-roam version.

Higher risk and high risk/possibly dependent drinkers who received the tunnelled version of the DBIU website were more likely to view the page on alcoholism, $X^{2}(1, N=47)=$ $13.81, p<.001$, than those who received the free-roam version.

Increasing risk and higher risk drinkers who received the tunnelled version of the DBIU website were more likely to view the page on creating a plan to cut down, $X^{2}(1, N=159)$ $=57.17, p<.001$, than those who received the free-roam version. They were also more likely to make a plan, $\mathrm{X}^{2}(1, \mathrm{~N}=159)=12.12, \mathrm{p}<.001(28 / 78 \mathrm{vs} .10 / 81)$, and made more plans, $\mathrm{t}(157)=3.68, \mathrm{p}<.001(\mathrm{Ms}=0.99, \mathrm{SD}=1.40$ vs. $0.31, \mathrm{SD}=0.87$ ), than those who received the free-roam version.

High risk/possibly dependent drinkers who received the tunnelled version of the DBIU website were more likely to view the page on what to do/who to contact about their drinking, $\mathrm{X}^{2}(1, \mathrm{~N}=21)=6.11, \mathrm{p}=.01$, than those who received the free-roam version. Part of this page also included an advice video on how to gain further support. Only one high risk/possibly dependent drinker watched the video (who received the tunnelled version of the website). As a result, it was not possible to formally test the effect of the intervention structure on this measure of engagement. 
Table 2. Pages visited by structure condition. Values are Ns.

\begin{tabular}{|l|l|l|l|l|}
\hline \multirow{2}{*}{} & \multicolumn{3}{l|}{ Structure Condition } \\
\cline { 2 - 5 } & \multicolumn{2}{l|}{ Free-roam } & No & \multicolumn{2}{l|}{ Yes } & No \\
\cline { 2 - 5 } & Yes & & 125 & 17 \\
\hline $\begin{array}{l}\text { What do units mean? } \\
\text { (All participants, } N=286)\end{array}$ & 75 & 67 & 125 \\
\hline $\begin{array}{l}\text { How does my drinking compare? } \\
\text { (All participants, } N=286)\end{array}$ & 41 & 101 & 120 & 24 \\
\hline $\begin{array}{l}\text { What are the risks? } \\
\text { (All participants, } N=286)\end{array}$ & 36 & 106 & 118 & 26 \\
\hline $\begin{array}{l}\text { What can I do next? } \\
\text { (Lower risk drinkers, } N=106)\end{array}$ & 19 & 35 & 44 & 8 \\
\hline $\begin{array}{l}\text { Am I an alcoholic? } \\
\text { (Higher risk and high risk/possibly } \\
\text { dependent drinkers, } N=47)\end{array}$ & 3 & 15 & 21 & 8 \\
\hline $\begin{array}{l}\text { Create a plan } \\
\text { (Increasing and higher risk drinkers, } N= \\
\text { 159) }\end{array}$ & 19 & 62 & 65 & 13 \\
\hline $\begin{array}{l}\text { Plan your next step (High risk/possibly } \\
\text { dependent drinkers, } N=21)\end{array}$ & 1 & 6 & 10 & 4 \\
\hline
\end{tabular}

\section{Reductions in alcohol consumption at one-month follow-up}

There was a significant main effect of time on weekly alcohol consumption (units), $\mathrm{F}(1,232)=19.78, \mathrm{p}<.001, \mathrm{~d}_{\mathrm{z}}=0.29$; consumption declined between baseline $(\mathrm{M}=$ 13.84, $S D=13.69)$ and one-month follow-up $(M=11.32, S D=11.11)$. The time $x$ structure, $F(1,232)=3.35, p=.07$, time $x$ planning, $F(1,232)=2.76, p=.10$, and time $x$ structure $x$ planning, $F(1,232)=0.76, p=.76$, interactions were all non-significant.

There was also significant main effect of time on the frequency of binge drinking, $F(1,232)=5.78, p=.02, d_{z}=0.16$; binge drinking declined between baseline $(M=0.81$, $\mathrm{SD}=1.78)$ and one-month follow-up $(\mathrm{M}=0.66, \mathrm{SD}=0.92)$. The time $\mathrm{x}$ structure, $\mathrm{F}(1,232)=1.97, \mathrm{p}=.16$, time $\times$ planning, $\mathrm{F}(1,232)=0.13, \mathrm{p}=.71$, and time $\mathrm{x}$ structure $\mathrm{x}$ planning, $F(1,232)=0.01, p=.94$, interactions were all non-significant.

These analyses were repeated for increasing risk and higher risk drinkers. As with the full sample, there was a significant main effect of time on weekly alcohol consumption, $F(1,129)=19.91, p<.001, d_{z}=0.39$, which declined between baseline $(M=17.59, S D=$ $12.47)$ and one-month follow-up $(M=14.23, S D=10.92)$. The time $x$ structure, $F(1,129)$ 
$=1.57, \mathrm{p}=.21$, time $\times$ planning, $\mathrm{F}(1,129)=2.01, \mathrm{p}=.16$, and time $\times$ structure $\times$ planning, $F(1,129)=0.00, p=.999$, interactions were all non-significant.

There was also significant main effect of time on the frequency of binge drinking for increasing risk and higher risk drinkers, $F(1,129)=7.54, p=.01, d_{z}=0.25$, which declined between baseline $(M=1.11, S D=1.16)$ and one-month follow-up $(M=0.85, S D$ $=0.96)$. The time $\times$ structure, $F(1,129)=0.75, p=.39$, time $\times$ planning, $F(1,129)=0.03, p$ $=.86$, and time $x$ structure $x$ planning, $F(1,129)=0.02, p=.89$, interactions were all nonsignificant.

\section{Reductions in alcohol consumption at six-month follow-up}

There was a significant main effect of time on weekly alcohol consumption (units), $\mathrm{F}(1,220)=8.54, \mathrm{p}=.004, \mathrm{~d}_{\mathrm{z}}=0.20$; consumption declined between baseline $(\mathrm{M}=14.74$, $S D=15.02)$ and six-month follow-up $(M=12.59, S D=12.85)$. The time $x$ structure, $\mathrm{F}(1,220)=0.25, \mathrm{p}=.62$, time $\mathrm{x}$ planning, $\mathrm{F}(1,220)=0.02, \mathrm{p}=.89$, and time $\mathrm{x}$ structure $\mathrm{x}$ planning, $F(1,220)=0.04, p=.85$, interactions were all non-significant.

There was also significant main effect of time on the frequency of binge drinking, $F(1,220)=8.14, p=.005, d_{z}=0.19$; binge drinking declined between baseline $(M=0.86$, $S D=1.21)$ and six-month follow-up $(M=0.68, S D=1.01)$. The time $x$ structure, $F(1,220)$ $=0.88, p=.35$, time $\times$ planning, $F(1,220)=0.76, p=.38$, and time $\times$ structure $\times$ planning, $F(1,220)=1.09, p=.30$, interactions were all non-significant.

There was also significant main effect of time on AUDIT scores, $F(1,220)=76.19, p<$ $.001, d_{z}=0.58$; AUDIT scores declined between baseline $(M=9.05, S D=5.46)$ and sixmonth follow-up $(M=6.86, S D=4.80)$. The time $x$ structure, $F(1,220)=2.20, p=.14$, time $\times$ planning, $F(1,220)=0.24, p=.63$, and time $\times$ structure $\times$ planning, $F(1,220)=$ $0.82, p=.37$, interactions were all non-significant.

These analyses were repeated for increasing risk and higher risk drinkers. As with the full sample, there was a significant main effect of time on weekly alcohol consumption, $F(1,123)=5.27, p=.02, d_{z}=0.19$, which declined between baseline $(M=18.09, S D=$ 13.56) and six-month follow-up $(M=15.96, S D=13.42)$. The time $x$ structure, $F(1,123)=$ 2.56, $\mathrm{p}=.14$, time $\times$ planning, $\mathrm{F}(1,123)=0.94, \mathrm{p}=.48$, and time $\times$ structure $\times$ planning, $F(1,123)=0.45, p=.50$, interactions were all non-significant.

There was also significant main effect of time on the frequency of binge drinking for increasing risk and higher risk drinkers, $F(1,123)=11.18, p=.001, d_{z}=0.30$, which declined between baseline $(M=1.13, S D=1.20)$ and six-month follow-up $(M=0.86, S D$ $=1.11)$. The time $\times$ structure, $F(1,123)=0.52, p=.47$, time $\times$ planning, $F(1,123)=0.50, p$ $=.48$, and time $x$ structure $x$ planning, $F(1,123)=0.02, p=.90$, interactions were all nonsignificant.

There was also significant main effect of time on AUDIT scores for increasing risk and higher risk drinkers, $\mathrm{F}(1,123)=75.08, \mathrm{p}<.001, \mathrm{~d}_{\mathrm{z}}=0.77$, which declined between baseline $(M=11.07, S D=3.15)$ and six-month follow-up $(M=8.38, S D=4.22)$. The time 
$x$ structure, $F(1,123)=1.58, p=.21$, time $x$ planning, $F(1,123)=2.16, p=.15$, and time $x$ structure $x$ planning, $F(1,123)=0.05, p=.83$, interactions were all non-significant.

\section{Attrition analyses}

There were no significant differences between participants who did and did not complete the one-month follow-up questionnaire in relation to staff/student status, $X^{2}(1, N=281)=$ 2.30, $p=.13$, gender, $X^{2}(1, N=281)=2.60, p=.11, p=.94$, age, $t(279)=1.88, p=.06$, nationality, $x^{2}(1, N=281)=0.05, p=.83$, ethnicity, $x^{2}(1, N=281)=0.44, p=.51$, and experimental condition, $x^{2}(3, N=281)=3.50, p=.32$. However, participants lost to onemonth follow-up consumed more alcohol, $\mathrm{t}(279)=3.96, \mathrm{p}<.001(\mathrm{Ms}=23.80, \mathrm{SD}=$ 22.64 vs. $13.84, S D=13.84$ ), engaged in binge drinking more frequently, $t(279)=2.02, p$ $=.04(\mathrm{Ms}=1.20, \mathrm{SD}=1.25$ vs. $0.681, \mathrm{SD}=1.18)$, and had higher AUDIT scores, $\mathrm{t}(279)$ $=4.00, p<.001(\mathrm{Ms}=12.33, \mathrm{SD}=7.44$ vs. $8.68, \mathrm{SD}=5.21)$, at baseline than those who remained in the study.

There were no significant differences between participants who did and did not complete the six-month follow-up questionnaire in relation to staff/student status, $x^{2}(1, N=282)=$ $0.53, p=.47$, gender, $X^{2}(1, N=278)=1.03, p=.31, p=.94$, age, $t(280)=1.60, p=.11$, nationality, $x^{2}(1, N=282)=2.37, p=.12$, ethnicity, $x^{2}(1, N=282)=0.43, p=.51$, experimental condition, $x^{2}(3, N=282)=0.80, p=.85$, frequency of binge drinking, $t(280)$ $=1.00, p=.32$, AUDIT scores, $t(280)=1.66, p=.10$, or risk level, $X^{2}(2, N=282)=3.83$, $p=.15$. However, participants lost to six-month follow-up consumed more alcohol at baseline, $\mathrm{t}(280)=2.34, \mathrm{p}=.02(\mathrm{Ms}=20.42, \mathrm{SD}=21.15$ vs. $14.74, \mathrm{SD}=15.02)$. 


\section{Discussion}

The present study had two main aims: (i) to test the effect of the structure of a brief online alcohol intervention on engagement and effectiveness and (ii) to test the effect of different planning instructions (on how to reduce alcohol consumption) on effectiveness. The study found that the way in which the content of intervention was structured had a significant effect on engagement, but not effectiveness, and that the type of planning task had a non-significant effect on effectiveness.

Considering the effect of the structure of the online intervention on engagement, participants who received the tunnelled version spent more time on the website, viewed more pages overall, and were more likely to view each of the specific information pages than those who viewed a free-roam version. These findings are in line with previous studies that have directly compared tunnelled and free-roam versions of the same online intervention using RCT designs. For example, Crutzen et al. (2012) found that participants who viewed a tunnelled version of a 12 page information website about hepatitis visited more pages and spent more time on the website than those who viewed a free-roam version. McClure et al. (2013) also found that participants who had access to the tunnelled version of an online smoking cessation intervention visited more pages and spent more time online than those who had access to the free-roam version. It is likely that many participants who are given autonomy over which pages to view, and in which order, in free-roam interventions use this autonomy to choose to view few, or no, pages. In contrast, tunnelling may encourage participants to continue to click through the pages as there are no obvious exit points (Fogg, 2003).

It is possible that although participants who receive tunnelled versions of an intervention spend more time and view more pages on the website, the nature of the engagement may be relative superficial (i.e., peripheral processing). Interestingly, although $83 \%$ of increasing risk and higher risk drinkers who received the tunnelled intervention viewed the page on creating a plan (compared to $23 \%$ who received the free-roam version), only half of these participants proceeded to actually make a plan to cut down. Similarly, although 10 out of the 11 high risk/possibly dependent drinkers who received the tunnelled intervention viewed the page describing options for further help (compared to 4 out of 10 who received the free-roam version), only one watched the optional advice video. These findings suggest that participants did not fully engage with the intervention. In contrast, Crutzen et al. (2012) reported that that the tunnelled intervention led to greater knowledge about hepatitis at one-month follow-up than the free-roam intervention, suggesting that participants who received the tunnelled version actively processed the information they viewed. Future work should seek to assess different aspects of engagement (Perski et al., 2017). In the present study only the extent of engagement was assessed (i.e., pages visited, time spent on website) rather than the depth of engagement (e.g., attention, depth of processing, retention), subjective experience (e.g., perceived ease of use, liking) or physiological (e.g., electrodermal activity) and psychophysical (e.g., eye tracking) reactions. 
In contrast to the effects on engagement, the structure of the intervention had no impact on reductions in alcohol consumption (i.e., units consumed, frequency of binge drinking) at one-month or six-month follow-up. This finding is consistent with McClure et al. (2014) who found that the structure of an online smoking cessation intervention had no impact on cessation rates at 12-month follow-up. It is possible that while a tunnelled online intervention increases the number of pages viewed, thereby exposing participants to more information that may help them to change their behaviour, participants who receive a free-roam version may, nonetheless, choose to view the most relevant information that helps them to change their behaviour. The fact that, overall, there were significant reductions in alcohol consumption between baseline and both one- and six-month followup, suggests that participants were able to view sufficient information to support reductions in drinking. Similarly, McClure et al. (2014) reported a 13.7\% abstinence rate at 12-month follow-up, which compares favourably with other smoking cessation interventions, including minimal self-help interventions (8.5\%), "quit-line" (telephone) counselling (12.7\%), and counselling alone (14.6\%) (Fiore et al., 2008).

Considering the effect of type of planning on effectiveness, it was found that there was no difference in reductions in alcohol consumption between increasing and higher risk drinkers who were instructed to choose between various behavioural strategies to cut down on their drinking and those who were instructed to form if-then plans linking highrisk situations with the behavioural strategies. This finding contrasts with previous research which has found that instructing participants to explicitly link situations and strategies leads to greater reductions in alcohol consumption than instructing participants to merely consider the situations and strategies, but not link them (Arden and Armitage, 2012; Armitage, 2015; Armitage and Arden, 2012). Similarly, studies that have tested the effect of forming if-then plans (i.e., implementation intentions) versus (no planning) control conditions have found they have significant effects on alcohol consumption (e.g., Hagger et al., 2012; Norman and Wrona-Clarke, 2016), although there are some null findings (e.g., Norman et al., 2018). As with the non-significant effect of structure on effectiveness, it is possible that participants viewed sufficient information to engender reductions in alcohol consumption, without the need for detailed if-then plans. It is noteworthy that all participants in the present study received feedback on their risk level (based on their AUDIT score). Feedback on performance has been identified as one of four key behaviour change techniques that are associated with greater reductions in alcohol consumption in a review and meta-analysis of online alcohol interventions (Black et al., 2016). Other behaviour change techniques that were associated with greater reductions in alcohol consumption included provision of normative information, prompting commitment and prompting review of goals. Future research should therefore seek to test the effectiveness of these behaviour change techniques, along with if-then plans (action planning), using experimental/factorial designs.

The brief online alcohol intervention (DBIU) was found to have a small effect on alcohol consumption and the frequency of binge drinking, irrespective of the structure and type of planning. Weekly alcohol consumption reduced by 2.58 units $\left(d_{z}=0.29\right)$ for all participants and 3.36 units $\left(d_{z}=0.39\right)$ for increasing risk and higher risk drinkers between baseline and one-month follow-up, and by 2.15 units $\left(d_{z}=0.20\right)$ for all participants and 2.13 units $\left(d_{z}=0.19\right)$ for increasing risk and higher risk drinkers, between baseline and 
six-month follow-up. The frequency of binge drinking also decreased by 0.15 times per week $\left(\mathrm{d}_{\mathrm{z}}=0.16\right)$ for all participants and 0.26 times per week $\left(\mathrm{d}_{\mathrm{z}}=0.25\right)$ for increasing risk and higher risk drinkers between baseline and one-month follow-up, and by 0.18 times per week $\left(d_{z}=0.19\right)$ for all participants and 0.27 times per week $\left(d_{z}=0.30\right)$ for increasing risk and higher risk drinkers between baseline and six-month follow-up. Larger sized reductions in AUDIT scores were observed between baseline and six-month followup for all participants $\left(d_{z}=0.58\right)$ and for increasing risk and higher risk drinkers $\left(d_{z}=\right.$ $0.77)$. These reductions compare favourably with the average effects of online alcohol interventions on total alcohol consumption $(d=0.15)$ and binge drinking $(d=0.07)$ (Black et al., 2016), although it should be noted that the present study did not include a no intervention control group so the effect of the intervention is based on pre-post scores and, as a result, may over-estimate the effect of the online intervention on alcohol consumption.

\section{Conclusion}

The findings of the present study suggest that brief online alcohol interventions may be used to encourage and support people to reduce their alcohol consumption. The present study found that a tunnelled version of the intervention resulted in participants spending more time and viewing more pages than a free-roam version. Brief online alcohol intervention should therefore employ a tunnelled structure in order to increase levels of engagement, although future work should assess other aspects of engagement (e.g., depth of processing) in addition to the extent of engagement. Neither the structure of the intervention or the type of planning task included in the intervention impacted on reductions in alcohol consumption. Future research should therefore seek to identify the "active ingredients" of brief online alcohol interventions in which the effectiveness of different behaviour change techniques are compared against each other using experimental/factorial designs. 


\section{References}

Arden, M.A., and Armitage, C.J. (2012). A volitional help sheet to reduce binge drinking in students: A randomized exploratory trial. Alcohol and Alcoholism, 47, 156-159. http://dx.doi.org/ 10.1093/alcalc/agr164

Armitage, C.J. (2015). Evidence that a volitional help sheet reduces alcohol consumption among smokers: A pilot randomized controlled trial. Behavior Therapy, 46, 342-349. doi: 10.1016/j.beth.2014.12.003

Armitage, C.J., and Arden, M.A. (2012). A volitional help sheet to reduce alcohol consumption in the general population: A field experiment. Prevention Science, 13, 3543. doi: $10.1007 / \mathrm{s} 11121-012-0291-4$

Babor, T.F., Higgins-Biddle, J.C., Saunders, J.B., and Nonteiro, M.G. (2001). The alcohol use disorders identification test. Geneva: World Health Organization.

Bertholet, N., Daeppen, J.B., Wietisbach, V., Fleming, M., and Burnand, B. (2005). Reduction of alcohol consumption by brief alcohol intervention in primary care. Archives of Internal Medicine, 165, 986-995. doi: 10.1001/archinte.165.9.986

Black, N., Mullan, B., and Sharpe, L. (2016). Computer-delivered interventions for reducing alcohol consumption: Meta-analysis and meta-regression using behaviour change techniques and theory. Health Psychology Review, 10, 341-357. doi:

10.1080/17437199.2016.1168268

Cancer Prevention Research Center. (2010). Alcohol: Processes of change. Retrieved from: https://web.uri.edu/cprc/alcohol-processes-of-change/

Crutzen, R., Cyr, D., and de Vries, N.K. (2012). The role of user control in adherence to and knowledge gained from a website: Randomized comparison between a tunneled version and a freedom-of-choice version. Journal of Medical Internet Research, 14, e45. doi: 10.2196/jmir.1922

Donkin, L., Christensen, H., Naismith, S.L., Neal, B., Hickie, I.B., and Glozier, N. (2011). A systematic review of the impact of adherence on the effectiveness of e-therapies. Journal of Medical Internet Research, 13, e52. doi: 10.2196/jmir.1772

Drinkaware. (2017). Unit \& calorie calculator. Retrieved from: https:// www.drinkaware.co.uk/understand-your-drinking/unit-calculator/

Fiore, M. C., Jaén, C. R., Baker, T. B., Bailey, W. C., Benowitz, et al. (2008). Treating tobacco use and dependence: 2008 update. Clinical practice guideline. Rockville, MD: U.S. Department of Healthand Human Services. Public Health Service.

Fogg, B.J. (2003). Persuasive technology: Using computers to change what we think and do. San Francisco: Morgan Kaufmann.

Fredericks, S., Martorella, G., and Catallo, C. (2015). A systematic review of web-based educational interventions. Clinical Nursing Research, 24, 91-113. doi:

$10.1177 / 1054773814522829$ 
Gollwitzer, P.M., and Sheeran, P. (2006). Implementation intentions and goal achievement: A meta-analysis of effects and processes. Advances in Experimental Social Psychology, 38. 69-119. doi:10.1013/S0065-2601(06)38002-1

Gomel, M.K., Saunders, J.B., Burns, L., Hardcastle, D.M., and Sumich M. (1994). Dissemination of early intervention for harmful alcohol consumption in general practice. Health Promotion Journal of Australia, 4, 65-69.

Hagger, M.S., Lonsdale, A., Koka, A., Hein, V., Pasi, H., Lintunen, T., and Chatzisarantis, N. L. D. (2012). An intervention to reduce alcohol consumption in undergraduate students using implementation intentions and mental simulations: A cross-national study. International Journal of Behavioral Medicine, 19, 82-96. doi:10.1007/s12529-011-9163-8

Kaner, E.F., Dickinson, H.O., Beyer, F.R., Campbell, F., Schlesinger, C., Heather, N., Saunders, J.B., Burnand, B., and Pienaar, E.D. (2007). Effectiveness of brief alcohol interventions in primary care populations. Cochrane Database of Systematic Reviews 2007, Issue 2. Art. No.: CD004148. doi: 10.1002/14651858.CD004148.pub3.

Kelders, S.M., Kok, R.N., Ossebaard, H.C., and van Gemert-Pijnen, J.E.W.C. (2012). Persuasive system design does matter: A systematic review of adherence to web-based interventions. Journal of Medical Internet Research, 14, e152. doi: 10.2196/jmir.2104

Maddock, J.E., LaForge, R.G., and Rossi, J.S. (2000). Short form of a situational temptation scale for heavy, episodic drinking. Journal of Substance Abuse, 11, 281-288. doi:10.1016/S0899-3289(00)00027-4

Manwaring, J.L., Bryson, S.W., Goldschmidt, A.B., Winzelberg, A.J., Luce, K.H., Cunning, D., Wilfley, D.E., and Taylor, C.B. (2008). Do adherence variables predict outcome in an online program for the prevention of eating disorders? Journal of Consulting and Clinical Psychology, 76, 341-346. doi: 10.1037/0022-006X.76.2.341

McClure, J.B., Peterson, D., Derry, H., Riggs, K., Saint-Johnson, J., Nair, V., An, L., and Shortreed, S.M. (2014). Exploring the "active ingredients" of an online smoking intervention: A randomized factorial trial. Nicotine and Tobacco Research, 16, 11291139. doi: $10.1093 /$ ntr/ntu057

McClure, J.B., Shortreed, S.M., Bogart, A., Derry, H., Riggs, K., Saint-Johnson, J., Nair, V., and An, L. (2013). The effect of program design on engagement with an internetbased smoking intervention: Randomized factorial trial. Journal of Medical Internet Research, 15, e69. doi: 10.2196/jmir.2508

Moyer, A., Finney, J.W., Swearingen, C.E., and Vergun, P. (2002). Brief interventions for alcohol problems: A meta-analytic review of controlled investigations in treatmentseeking and non-treatment-seeking populations. Addiction, 97, 279-292. doi: 10.1046/j.1360-0443.2002.00018.x

Norman, P., Cameron, D., Epton, T., Webb, T.L., Harris, P.R., Millings, A., and Sheeran, P. (2018). A randomised controlled trial of a brief online intervention to reduce alcohol consumption in new university students: Combining self-affirmation, theory of planned behaviour messages, and implementation intentions. British Journal of Health Psychology, 23, 108-127. doi: 10.1111/bjhp.12277 
Norman, P., and Wrona-Clarke, A. (2016). Combining self-affirmation and implementation intentions to reduce heavy episodic drinking in university students. Psychology of Addictive Behaviors, 30, 434-441. doi: 10.1037/adb0000144

Perski, O., Blandford, A., West, R., and Michie, S. (2017). Conceptualising engagement with digital behaviour change interventions: A systematic review using principles from critical interpretive synthesis. Translational Behavioral Medicine, 7, 254-267. doi: 10.1007/s13142-016-0453-1

Public Health England. (2017). Treatment outcomes and effectiveness: Treatment outcomes profile (TOP). Retrieved from: http://www.nta.nhs.uk/top-brief.aspx/

Sobell, L.C., and Sobell, M.B. (1992). Timeline follow-back: A technique for assessing self-reported alcohol consumption. In: Litten RZ, Allen JP, eds. Measuring alcohol consumption. Totowa, NJ; Human Press, pp. 41-72.

Webb, T.L., Joseph, J., Yardley, L., and Michie, S. (2010). Using the internet to promote health behavior change: A systematic review and meta-analysis of the impact of theoretical basis, use of behavior change techniques, and mode of delivery on efficacy. Journal of Medical Internet Research, 12, e4. doi:10.2196/jmir.1376 


\section{Appendix - Example screenshots of the DBIU website}

Screenshot 1. DBIU home page

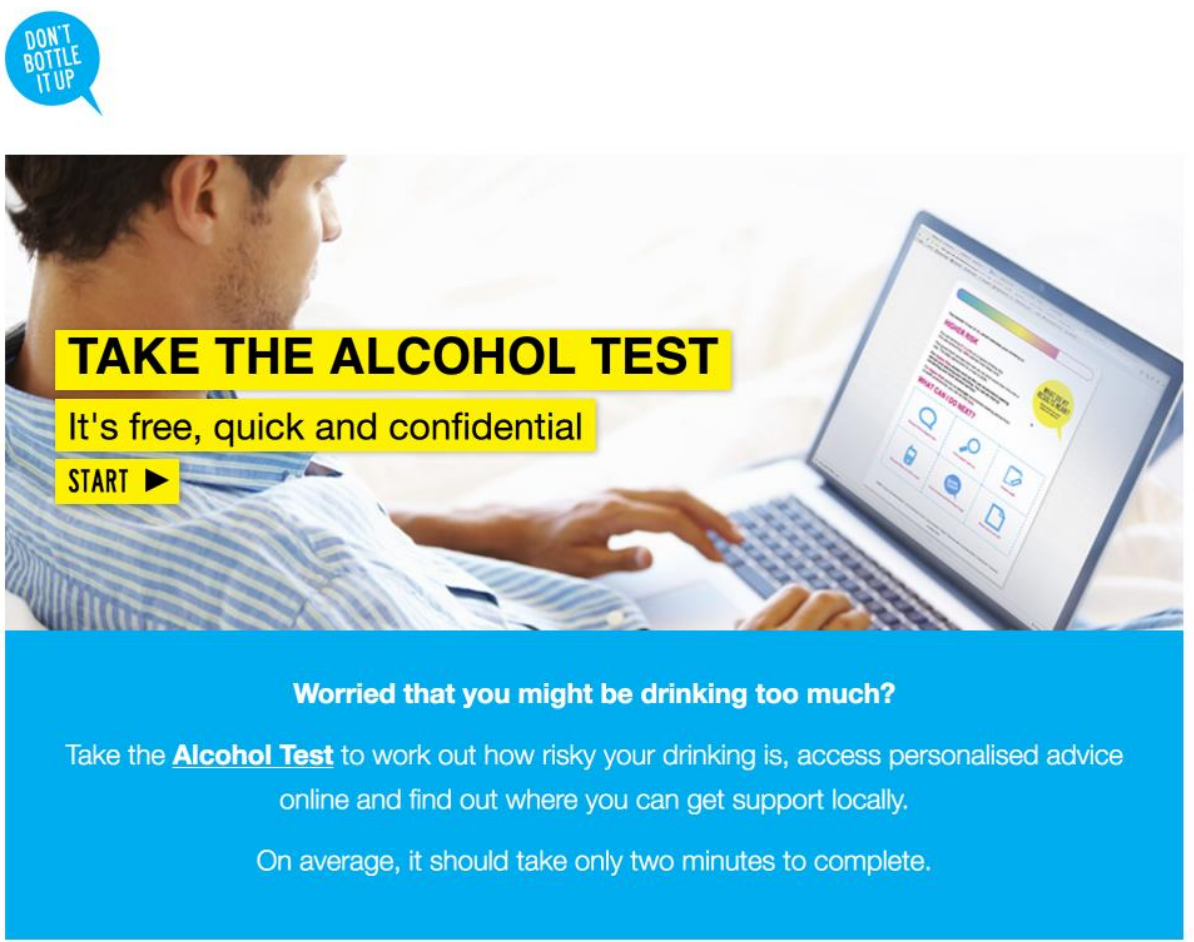

Screenshot 2. Feedback page (e.g. Higher risk drinker)

\section{YOU ARE A HIGHER RISK DRINKER}

\section{You scored 16 out of 40 , which indicates your drinking is: Higher Risk}

For Higher Risk scores such as this we recommend seeking advice and reducing your drinking. You can do that by contacting your local alcohol service:

To find about about support for alcohol use in Sheffield, click here

You are drinking $\mathbf{5 . 5}$ units on a typical drinking day. You are consuming $\mathbf{7 7 0}$ calories from these units.

The government advises that women do not drink more than 14 units per week, with at least two days alcohol free. The suggested daily calorie limit for an average woman is 2000 (this can vary depending on age, metabolism and levels of physical activity).

\section{WHAT CAN I DO NEXT?}

Read more about YOUR drinking

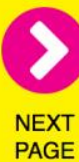




\section{Screenshot 3. What do units mean? (e.g. Higher risk drinker)}

\section{WHAT DO UNITS MEAN?}

Your score suggest that you are regularly drinking double the recommended limits for men.

Don't drink more than 3-4 units per day.

- Have at least two alcohol-free days every week.

- Remember the number of units in the drinks you regularly drink, so you can keep track and perhaps try cutting down slowly.

The unit guide below will help:

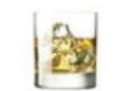

2 Units

Double $50 \mathrm{ml}$ measure of spirits $(40 \%)$

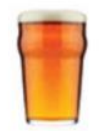

1 Unit

$1 / 2$ pint "regular" beer, lager or cider $(4 \%)$

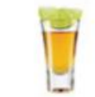

$$
1 \text { Unit }
$$

Single $25 \mathrm{ml}$ measure of spirits $(40 \%)$

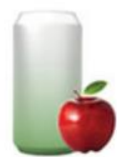

$$
2 \text { Units }
$$

$440 \mathrm{ml}$ can of "regular" lager or cider $(4 \%)$
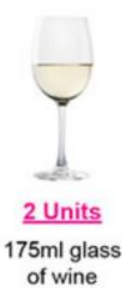

of wine

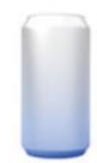

4 Units

$440 \mathrm{ml}$ can of "super strength" lager or cider

$(9 \%)$

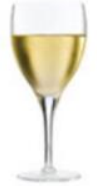

$$
3 \text { Units }
$$$$
250 \mathrm{ml} \text { glass }
$$$$
\text { of wine }
$$$$
(12 \%)
$$

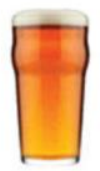

2 Units

Pint of "regular" beer, lager or cider $(4 \%)$

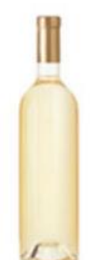

9 Units $750 \mathrm{ml}$ bottle of wine $(12 \%)$

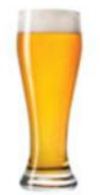

$$
3 \text { Units }
$$$$
\text { Pint of }
$$$$
\text { "premium" beer. }
$$
lager or cider

$(5 \%)$

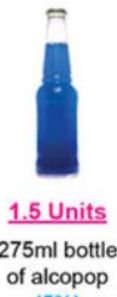

$(5 \%)$

Screenshot 4. How does my drinking compare? (e.g. Higher risk drinker)

\section{HOW DOES MY DRINKING COMPARE?}

Your score suggests that you are at Higher Risk of harm as a result of your drinking.

Take a look at the pie chart: whilst $78 \%$ of people in the UK stay within the recommended limits, roughly $\mathbf{5 \%}$ drink at Higher Risk.

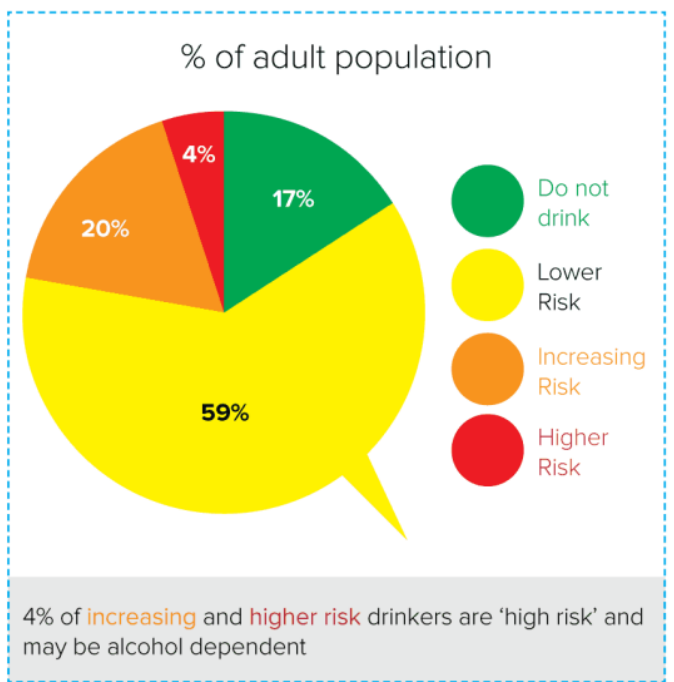




\section{Screenshot 5. What are the risks? (e.g. Higher risk drinker)}

\section{WHAT ARE THE RISKS?}

Alcohol affects all the parts and systems of your body, and it plays a role in more than sixty different medical conditions.

As a Higher Risk drinker, you might have already experienced, or may experience in the future, some problems linked to alcohol, such as:

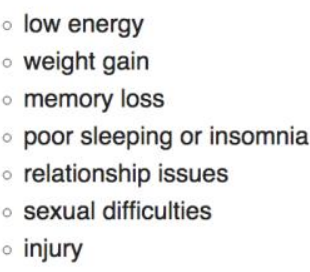

In the long-term, Higher Risk drinking significantly raises the risk of:

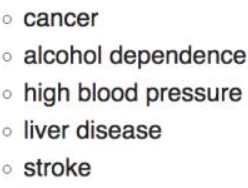

By drinking at Higher Risk levels:

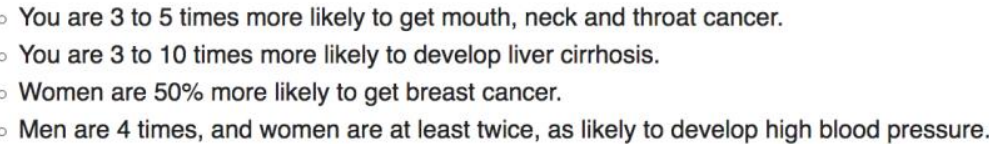

You are 3 to 5 times more likely to get mouth, neck and throat cancer. You are 3 to 10 times more likely to develop liver cirrhosis.

Women are $50 \%$ more likely to get breast cancer.

Men are 4 times, and women are at least twice, as likely to develop high blood pressure.

You are twice as likely to suffer from irregular heartbeat.

\section{Drinking and driving}

We can't tell you how much alcohol you can drink and remain within the strict drink driving limit. How alcohol affects your body depends on your weight, age, sex, metabolism, what you have drunk and how much, what you've eaten and how stressed you are. Even small amounts of alcohol can affect your ability to drive safely, so never drink and drive.

\section{Alcohol and pregnancy}

If you are pregnant, you shouldn't drink alcohol at all. Even small amounts of alcohol can damage your baby, causing birth defects, learning difficulties, miscarriage or stillbirth.

\section{Screenshot 6. What can I do next? (e.g. Lower risk drinker)}

\section{WHAT CAN I DO NEXT?}

Your score suggests that you don't need to make any changes to your drinking. Stay aware of how often and how much you are drinking though to make sure that your drinking doesn't become more risky. 


\section{Screenshot 7. Am I an alcoholic? (e.g. Higher risk drinker)}

\section{AM I AN ALCOHOLIC?}

Your score suggests that you are at risk of becoming alcohol dependent.

Some people use the phrase "alcoholic"; others talk about "alcohol dependence." Whatever you choose to call it, drinking at Higher Risk levels is associated with negative effects on your physical and mental health and well-being, such as alcohol dependence.

Alcohol dependence syndrome-a condition that affects $4 \%$ of adults in the UK-covers a range of symptoms, including:

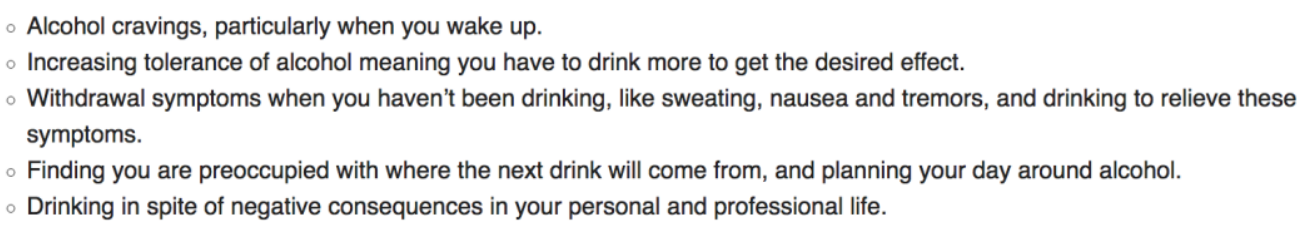

Dependent drinking is associated with reduced life expectancy, difficulties with relationships and work, and deteriorating health and well-being.

We strongly recommend that you talk to a health professional about how to cut down safely.

\section{Screenshot 8. Plan your next step (High risk/possibly dependent drinkers)}

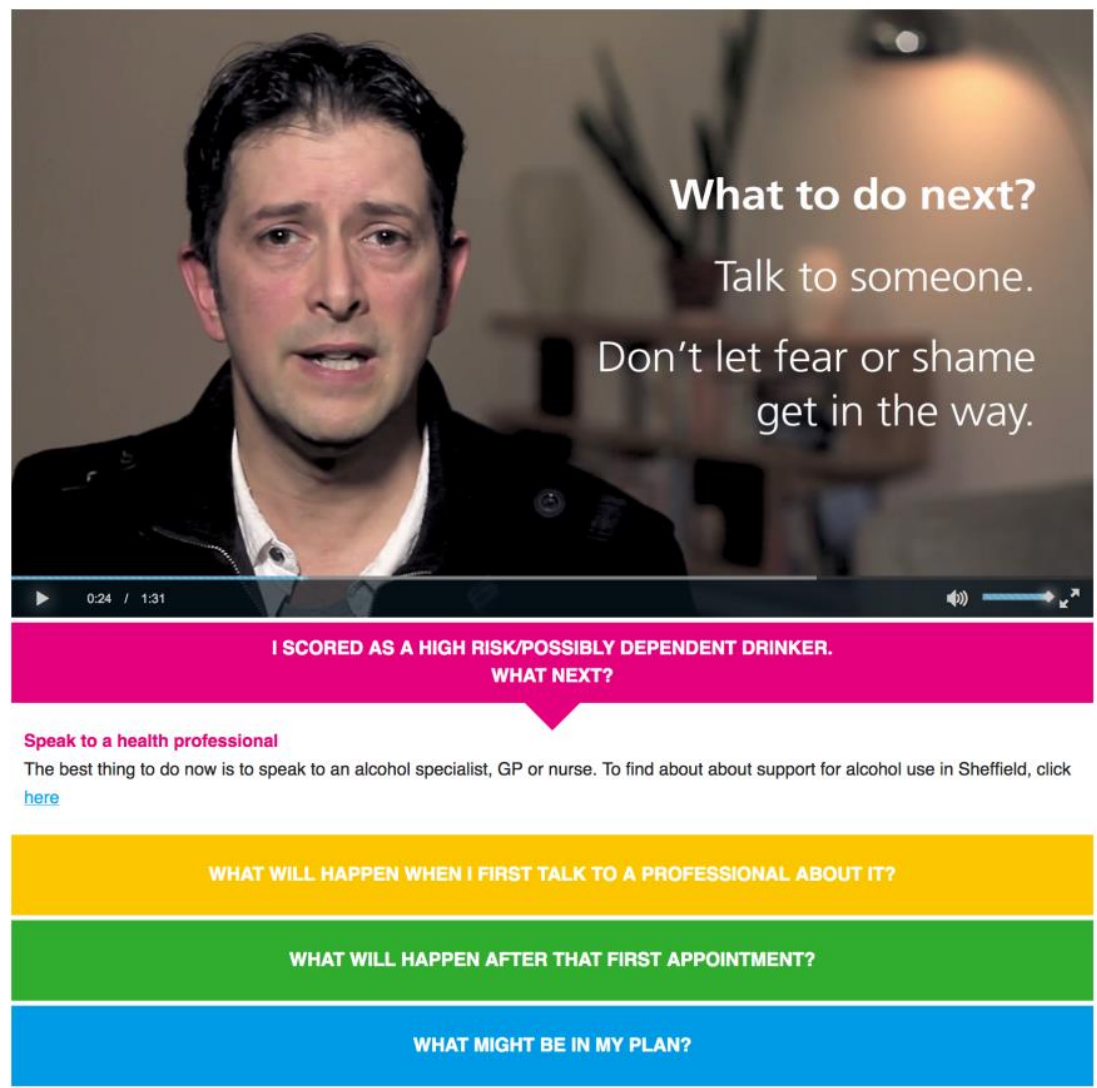




\section{Screenshot 9. Create a plan: Strategies (Higher risk and increasing risk drinkers)}

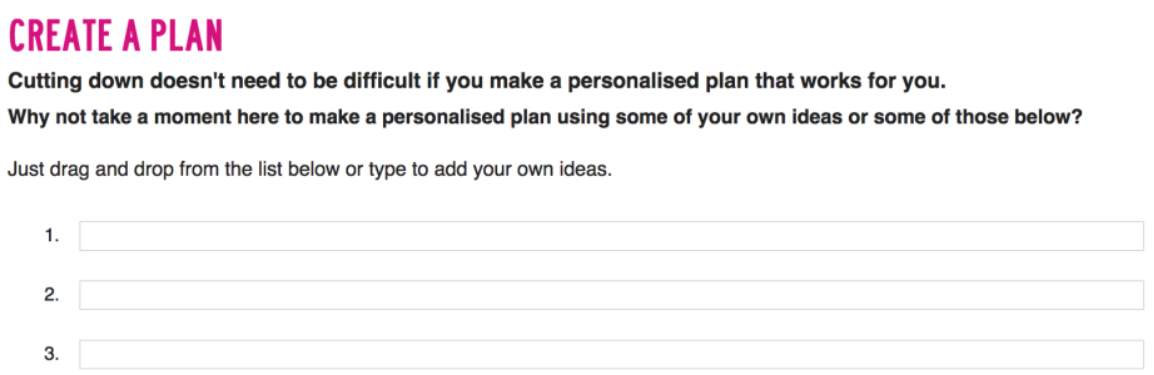

Only you can make the decision to cut down and you will know how best to do it. When thinking about cutting down, here are a few ideas that might be useful:

\begin{tabular}{l} 
Plan other activities and tasks \\
Explore other Interests, such as cinema, theatre, art, and music \\
\hline Have a workout or exercise \\
\hline Avoid going to the pub \\
Have my first drink after starting to eat \\
\hline Quench my thirst with non-alcohol drinks before and in between alcoholic drinks \\
\hline Avoid drinking in rounds or large groups \\
\hline Switch to lower strength (i.e. from $5 \%$ to $4 \%$ drinks \\
\hline Set myself a limit and stick to it \\
\hline Avoid or limit time spent with "heavy" drinking friends
\end{tabular}

\section{Screenshot 10. Create a plan: If-then plans (Higher risk and increasing risk drinkers)}

\section{CREATE A PLAN}

Cutting down doesn't need to be difficult if you make a personalised plan that works for you.

Why not take a moment here to make a personalised plan using some of you own ideas or some of those below?

You are more likely to cut down if you think about situations where you could cut down (or are tempted to drink heavily) and then think about things you can do in these situations. For example, you could say to yourself... "If I come home late from work and I'm tempted to have a glass of wine, then I will have a fruit smoothie instead" Or you could say... "If I feel under pressure to drink heavily when out with friends, then I will remind myself that I have important things to do tomorrow".

Now make your own plan of how you will cut down.

Think about the situations where you could cut down or where you might drink heavily - some situations are listed below or there may be other situations that you can think of. Then think about what you can do in these situations to cut down - some ideas of what you can do are listed below or there may be other ideas that you can think of.

Just drag and drop from the list below, or type in your own ideas, to make your own plans to cut down..

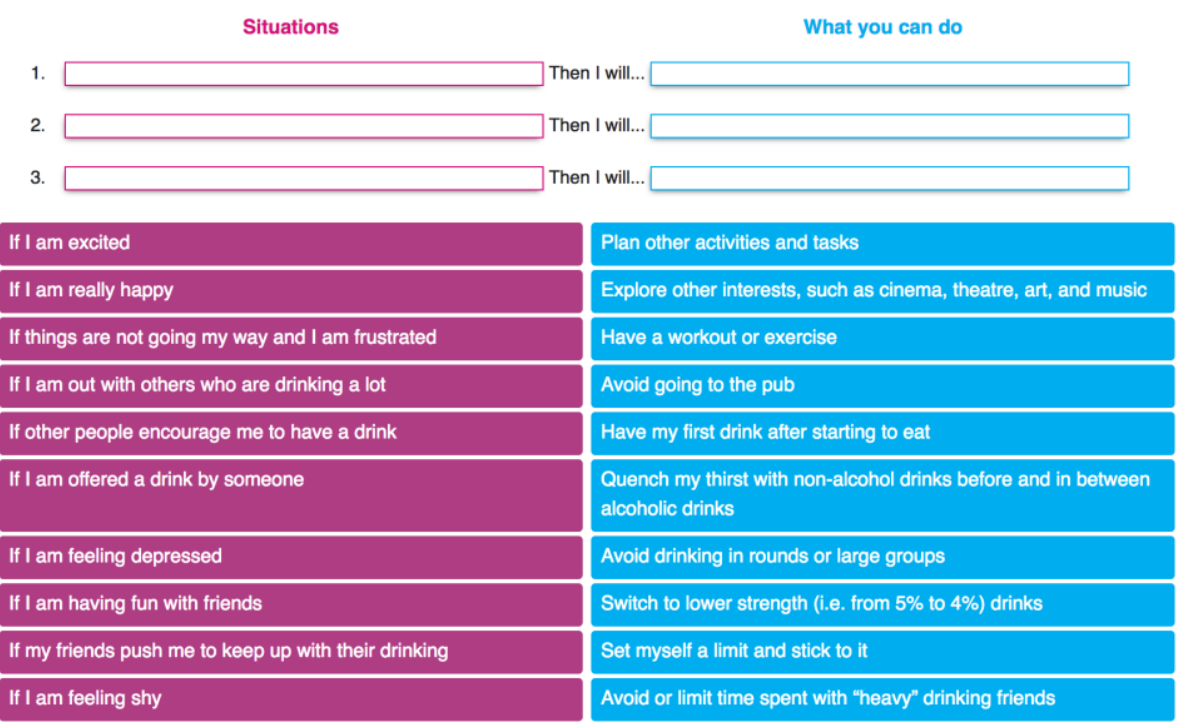

